CERN-TH/99-383

\title{
IRREVERSIBILITY AND HIGHER-SPIN CONFORMAL FIELD THEORY
}

\author{
Damiano Anselmi \\ CERN, Theory Division, CH-1211, Geneva 23, Switzerland
}

\begin{abstract}
I discuss the properties of the central charges $c$ and $a$ for higher-derivative and higher-spin theories (spin 2 included). Ordinary gravity does not admit a straightforward identification of $c$ and $a$ in the trace anomaly, because it is not conformal. On the other hand, higherderivative theories can be conformal, but have negative $c$ and $a$. A third possibility is to consider higher-spin conformal field theories. They are not unitary, but have a variety of interesting properties. Bosonic conformal tensors have a positive-definite action, equal to the square of a field strength, and a higher-derivative gauge invariance. There exists a conserved spin-2 current (not the canonical stress tensor) defining positive central charges $c$ and $a$. I calculate the values of $c$ and $a$ and study the operator-product structure. Higherspin conformal spinors have no gauge invariance, admit a standard definition of $c$ and $a$ and can be coupled to Abelian and non-Abelian gauge fields in a renormalizable way. At the quantum level, they contribute to the one-loop beta function with the same sign as ordinary matter, admit a conformal window and non-trivial interacting fixed points. There are composite operators of high spin and low dimension, which violate the Ferrara-Gatto-Grillo theorem. Finally, other theories, such as conformal antisymmetric tensors, exhibit more severe internal problems. This research is motivated by the idea that fundamental quantum field theories should be renormalization-group (RG) interpolations between ultraviolet and infrared conformal fixed points, and quantum irreversibility should be a general principle of nature.
\end{abstract}

December, 1999 - CERN-TH/99-383 


\section{Statement of the problem}

In the approach to quantum field theory as a radiative interpolation between pairs of conformal fixed points (see [1] for a brief survey) important physical information is given by the values of the central charges $c$ and $a$ at the fixed points and their dependence on the energy scale, in particular the differences $c_{\mathrm{UV}}-c_{\mathrm{IR}}$ and $a_{\mathrm{UV}}-a_{\mathrm{IR}}$. In a conformal theory, $c$ and $a$ are defined by the trace anomaly computed in the presence of an external gravitational field. The quantity $c$ multiplies the square of the Weyl tensor $W_{\mu \nu \rho \sigma}^{2}$ and is the coefficient of the two-point function of the stress tensor. The quantity a multiplies the Euler density $\mathrm{G}_{4}=\varepsilon_{\mu \nu \rho \sigma} \varepsilon_{\alpha \beta \gamma \delta} R^{\mu \nu \alpha \beta} R^{\rho \sigma \gamma \delta}$. A third term, $\square R$, is multiplied by a coefficient $a^{\prime}$ :

$$
\Theta=\frac{1}{(4 \pi)^{2}}\left[-c W^{2}+\frac{a}{4} \mathrm{G}_{4}-\frac{2}{3} a^{\prime} \square R\right] .
$$

In various UV-free supersymmetric models it is possible to compute $c$ and $a$ exactly in the IR limit [2]. In classically conformal renormalizable theories, a simple non-perturbative formula for the total RG flow of $a$ [3] holds, which shows the phenomenon of quantum irreversibility (the inequality $a_{\mathrm{UV}} \geq a_{\mathrm{IR}}$ ) [3, 4, 旬. The quantity $a$ is interpreted as a counter of the massless degrees of freedom.

A natural problem is to study the generalization of these results to higher-spin fields, gravity in particular. It is not straightforward to define the central charges $c$ and $a$ for gravity or higherspin fields, because they are not conformal. The trace anomaly for higher-spin fields was studied by Christensen and Duff [6] long ago and contains the square of the Ricci curvature $R$ :

$$
\Theta=\frac{1}{(4 \pi)^{2}}\left[-c W^{2}+\frac{a}{4} \mathrm{G}_{4}+\zeta R^{2}-\frac{2}{3} a^{\prime} \square R\right],
$$

a sign that the field is not conformal. The definition of $c$ and $a$ from the trace anomaly is unambiguous only if there is no such term. Explicitly, for spin $3 / 2$ and spin 2 we find, from table II of ref. [6] (omitting the $\square R$-term):

$$
\begin{aligned}
\Theta_{3 / 2} & =\frac{1}{360(4 \pi)^{2}}\left[255 W^{2}-22 \frac{\mathrm{G}_{4}}{4}+\frac{61}{2} R^{2}\right], \\
\Theta_{2} & =\frac{1}{360(4 \pi)^{2}}\left[-297 W^{2}-127 \frac{\mathrm{G}_{4}}{4}-\frac{717}{2} R^{2}\right] .
\end{aligned}
$$

We see that the graviton and gravitino contributions to the $R^{2}$-term have opposite signs. This means that a suitable combination of gravitons and gravitinos can cancel the $R^{2}$-term and might define a good higher-spin generalization of conformal field theory. It would be interesting to have a comprehensive list of the theories with vanishing $R^{2}$-term, starting from the analysis of Christensen and Duff, and understand if they can be considered conformal for all purposes. The disappearance of the $R^{2}$-term is a necessary condition, but might not be sufficient. Observe, however, that it is not simple to construct manifestly gauge-invariant stress tensors for higherspin fields and the $R^{2}$-term might depend on the definition, the gauge-fixing or the scheme 
choice. On the other hand, the conformal higher-spin fields, which are studied in this paper, have better properties in connection with these issues.

If sensible definitions of $c$ and $a$ are not separately available, it might be interesting to look for an appropriate generalization of the subclass of conformal field theories that have $c=a$. These theories share various properties with two-dimensional conformal field theory [5]. We see that the difference $c-a$ multiplies the unique term containing the Riemann tensor in the trace anomaly, $R_{\mu \nu \rho \sigma}^{2}$ in the trace anomaly. An appropriate definition of $c=a$ theories of gravitons and gravitinos, or higher-spin fields in general, might include the theories whose trace anomaly contains only $R_{\mu \nu}^{2}, R^{2}$ and $\square R$, but not $R_{\mu \nu \rho \sigma}^{2}$. In arbitrary even dimensions the trace anomaly of the $c=a$ theories contains the "minimal amount" of Riemann tensors, as shown in ref. [5]. However, the $c=a$ theories, certainly mathematically interesting, appear to be phenomenologically disfavoured (sect. 1.2).

In general, when do we have sensible definitions of $c$ and $a$ ? We first demand that the classical theory be conformal, so that no $R^{2}$-term is present in the trace anomaly. Secondly, we would like that $c$ and $a$ be both positive. Negative central charges represent a severe violation of unitarity. The purpose of this paper is to explore a large variety of theories, old and new, in this spirit. We begin with higher-derivative theories (section 2) and show that they have negative $c$ and $a$. We continue by classifying the higher-spin conformal theories. Fermions admit a straightforward coupling to gravity and gauge fields, so that in this case our program can be carried over to the end. There is evidence of a conformal window and that these theories obey the irreversibility property. On the other hand, higher-spin conformal bosons do not admit a straightforward coupling to gravity. Nevertheless, I show that there exists a suitable spin-2 current that has all the features to define appropriate $c$ and $a$. I compute their values in a simple case and show that they are positive.

Higher-spin conformal field theories are not unitary [7], but have a number of interesting features (of which conformal invariance is just the most important), which makes them interesting either as a laboratory for investigations in the spirit of [1] and the questions raised above, or for the description of physical phenomena in limited energy ranges. In some cases, they have a positive-definite action in the Euclidean framework. Symmetric conformal tensors have a higher-derivative gauge symmetry, which is investigated in detail. It is the unique gauge transformation compatible with the conformal symmetry. Moreover, these theories admit proper definitions of field strengths, dual field strengths, Chern-Simons forms, topological invariants, etc.

The non-unitarity of these theories can show up in the negative sign of $c$ or $a$, as remarked above. But even when $c$ and $a$ are positive, there might be effects on the anomalous dimensions of the operators generated by the OPE of two stress tensors. For example, the Ferrara-GattoGrillo theorem [9] states that, in a unitary theory, primary composite operators with spin $s$ should have a total dimension greater than or equal to $2+s$. This theorem is here manifestly violated. Indeed, the higher-derivative gauge invariance allows for "multiply-conserved" currents with dimensions $\Delta=2+s, 1+s, \ldots, 3$. Some of these operators will be constructed explicitly. 
There have been earlier works on conformal field equations of spin-2 [12, 13, 14, 15, 16] and spin-3/2 15] fields. These theories are particular cases of the ones presented below. To my knowledge the relationship between conformal invariance and higher-derivative gauge invariance was not known. Recently, related theories have attracted some interest in the domain of nuclear physics, where the purpose is to account for the hadronic resonances, such as the spin-3/2 $\Delta(1232)$ [17, 18]. I believe that the properties outlined here might be useful in this domain, at least in a definite energy range. The hope is that dynamical effects might make these theories consistent at low energies, thanks to quantum irreversibility itself (the ghosts might disappear above the Planck length, far before the physically observable degrees of freedom) or to a generalization of the Nachtmann theorem [8].

Finally, I remark that the study of higher-spin conformal field theory is in some sense complementary to the Fradkin-Vasiliev higher-spin field theory [10], which is unitary, but not conformal.

The plan of the paper is as follows. Before entering the technical part, I explain in sect. 1.1 the reasons why it is physically attractive to investigate higher-spin theories focusing on $c$ and $a$ and the irreversibility. Section 2 contains observations about higher-derivatives conformal field theories and the rest of the paper is about higher-spin conformal field theories. I present the bosonic conformal fields in section 3, the fermionic fields in section 5. Section 4 is devoted to a detailed analysis of the spin-2 field, with computations of $c$ and $a$ and a study of the operator-product $(\mathrm{OPE})$ structure. In section 5 the contribution to the gauge beta function from conformal spin-3/2 matter fermions is computed. I work in the Euclidean framework throughout this paper.

\subsection{Other motivations for the approach suggested here}

The search for a consistent formulation of higher-spin fields is difficult, and it might help to establish some general guidelines. This section is devoted to explaining why the properties of the central charges $c$ and $a$ and quantum irreversibility might be good for this. The ideas contained here should be meant as a work hypothesis. They apply to renormalizable, unitary theories of fields with spin $0,1 / 2$ and 1 , but their generalization to higher-spin fields, gravity in particular, is not evident. Yet, it is a bit uncomfortable that fields of spin $0,1 / 2$ and 1 have such a different status from fields of higher spin and a "unified" description would be desirable.

The two principles underlying the proposed approach are: $(i)$ a "conformal hypothesis", saying that every quantum field theory describing the phenomena of nature should be a renormalization-group interpolation between a UV conformal field theory and an IR conformal field theory; and $(i i)$ the irreversibility principle, stating that all fundamental theories of nature should be quantum irreversible, i.e. satisfy $a_{\mathrm{UV}} \geq a_{\mathrm{IR}}$.

We can distinguish between a strong form of the conformal hypothesis, when the classical action is conformal-invariant and all dimensionful parameters descend from the RG scale $\mu$, and a weak form of this hypothesis, when Newton's constant and, eventually, other nonrenormalizable parameters, descend from $\mu$, like $\Lambda_{\mathrm{QCD}}$, but the classical action is allowed to 
contain super-renormalizable terms and masses and be conformal only in their absence. Massless QCD obeys the strong form of the conformal hypothesis (the masses of hadrons are proportional to $\Lambda_{\mathrm{QCD}}$ and therefore descend from $\mu$ ), while massive QCD obeys the weak form of the conformal hypothesis (the pion masses and corrections to hadron masses are generated by the quark masses, i.e. they do not descend from $\mu$ ).

Theories obeying the strong conformal hypothesis fall under the treatment of refs. [3, 4] and admit a general formula expressing the difference $a_{\mathrm{UV}}-a_{\mathrm{IR}}$ [3] as the invariant area of the graph of the beta function. Theories obeying the weak conformal hypothesis also satisfy the inequality $a_{\mathrm{UV}} \geq a_{\mathrm{IR}}$ 円 , but the actual value of $a_{\mathrm{UV}}-a_{\mathrm{IR}}$ is not measured by the same formula [5]. These two cases can be assimilated to each other at the quantitative level when $c=a$ [5]. It is not clear at present how to link these two forms of irreversibility in the general case $c \neq a$, but presumably there is no need to either, since the effects of masses can usually be included straightforwardly [11. Observe that the irreversibility property implies that a perturbative formulation of quantum field theory is meaningful only from the UV. Since degrees of freedom are lost from the UV to the IR, a quantum field theory formulated from the IR (such as in IR-free theories) should be plagued by inconsistencies or be trivial ${ }^{2}$.

If the ultimate theory of the world falls into one of the two classes mentioned here, then the theories not obeying the (strong or weak) conformal hypothesis should be viewed as low-energy effective theories descending from high-energy fundamental theories that obey the conformal hypothesis. In particular, any non-renormalizable interaction, and gravity in particular, should be a low-energy effect. Observe that, if it were not so, non-renormalizable terms, admitting that we can make sense out of them without additional fields in the theory, are expected to violate the irreversibility principle. For example, a term $\varphi^{6} / \mathrm{m}^{2}$ forces the field $\varphi$ to vanish identically in the UV limit $m \rightarrow 0$ and leaves the IR unchanged. In certain supersymmetric theories 11] where some treatment of non-renormalizable terms has been claimed to be meaningful in the context of the so-called electric-magnetic duality, it has been found that non-renormalizable terms do violate the irreversibility statement (see section 6 of [1]]).

Finally, a special place is occupied by the " $c=a$ flows", i.e. flows that connect UV and IR fixed points in such a way that the difference $c-a$ remains constant (not necessarily zero), or at least $a_{\mathrm{UV}}-a_{\mathrm{IR}}=c_{\mathrm{UV}}-c_{\mathrm{IR}}$. The fixed points might or not have $c=a$. For example, taking a direct product between a $c=a$ flow connecting two $c=a$ fixed points and a free-field theory, we can obtain a $c=a$ flow connecting $c \neq a$ fixed points. These flows also fall under the treatment of [5]. In such cases dimensionless parameters can be assimilated to dimensionful coupling constants, for example masses and, conceivably, also the Newton constant, even if they are not induced by $\mu$. The weak point of these theories is that they are phenomenologically disfavoured. A necessary condition fot a $c=a$ flow is obtained by comparing the values of $c$

\footnotetext{
${ }^{1}$ A mass term $\frac{1}{2} m^{2} \varphi^{2}$ or, in general, super-renormalizable terms, has the effect of killing degrees of freedom in the IR, while the UV is left unchanged [19]. Roughly, this happens because the limit $m \rightarrow \infty$ is compatible only with $\varphi \equiv 0$.

${ }^{2}$ For example, the Landau poles in QED, the non-perturbative triviality of the $\lambda \phi^{4}$-theory, the perturbative non-renormalizability of quantum gravity. There is a good amount of evidence that QCD, instead, is fully consistent.
} 
and $a$ at energies admitting (approximate) free-field descriptions. Using the free-field values $c=\left(N_{s}+6 N_{f}+12 N_{v}\right) / 120$ and $a=\left(N_{s}+11 N_{f}+62 N_{v}\right) / 360, N_{s, f, v}$ being the numbers of real scalars, Dirac fermions and vectors, respectively, the differences between the numbers of spin-0, 1/2, 1 fields at two such energies are related in this case by the formula

$$
2 \Delta N_{s}+7 \Delta N_{f}=26 \Delta N_{v}
$$

Comparing the UV and IR limits of massless QCD, we find $\Delta N_{s}=-n_{f}^{2}+1, \Delta N_{f}=N_{c} n_{f}$, $\Delta N_{v}=N_{c}^{2}-1$, where $N_{c}$ is the number of colours and $n_{f}$ is the number of quark flavours. It is easy to check that the condition has no solution. Similarly, the spectra of the known low-energy physics do not appear to obey (1.2). For example, in the IR we can neglect the electron, but we have to include it at energies comparable with its mass. Formula (1.2) implies that as soon as the electron, or other fermions, becomes important, also vector fields should appear. There is no evidence of such a behaviour in nature. Finally, neither the Standard Model nor QCD have $c=a$.

We have analysed various phenomena of ordinary theories that might suggest valid guidelines for possible generalizations to new theories and gravity. These are, in summary, the reasons why I think that it is interesting to investigate higher-derivative theories and higher-spin conformal field theories starting from the properties of $c$ and $a$. We are now ready for the more technical part. We first study higher-derivative theories and then higher-spin conformal theories.

\section{Higher-derivative conformal field theories}

I begin by studying $c$ and $a$ in higher-derivative conformal field theories. The free higherderivative scalar field is interesting because it corresponds to the induced action for the conformal factor $\phi$ in an ordinary renormalizable theory and is described by the lagrangian

$$
S=\frac{1}{2} \int \mathrm{d}^{4} x \sqrt{g}\left[\phi \Delta_{4} \phi+\frac{Q}{16 \pi} \tilde{\mathrm{G}}_{4} \phi\right],
$$

where $\Delta_{4}=\square^{2}+2 R^{\mu \nu} \nabla_{\mu} \nabla_{\nu}-\frac{2}{3} R \square+\frac{1}{3}\left(\nabla^{\mu} R\right) \nabla_{\mu}$ [20, 21] and $\tilde{\mathrm{G}}_{4}=\mathrm{G}_{4}-\frac{8}{3} \square R$ is the "pondered" Euler density [ [⿴囗十 . Here $Q$ is a background charge. It does affect the induced effective action for the gravitational field, but its contribution is not of anomalous origin (see below). I keep $Q$ non-zero to show that it cannot be used to make either $c$ or $a$ positive.

The theory (2.3) has been studied in refs. [21, 22] and is the four-dimensional analogue of the free two-dimensional scalar field. Non-unitarity is evident from the fact that $c$ and $a$ are negative:

$$
c=-\frac{1}{15}, \quad a=-\frac{7}{90}-Q^{2} .
$$

\footnotetext{
${ }^{3}$ We can check it in the free-field limits. QED has $N_{v}=1, N_{f}=1$ and $c-a=-37 / 360$. Massless QCD has $N_{v}=8, N_{f}=18$ and $c-a=-41 / 180$. The electroweak theory has $N_{v}=4, N_{f}=9 / 2, N_{s}=4$ and $c-a=-43 / 180$. The Standard Model has $c-a=-293 / 720$. We see that $c-a$ is always negative, which means that there are many vector fields.
} 
The values at $Q=0$ can be read from [20, 21] and are the contributions coming from the determinant of $\Delta_{4}$. The contribution of the background charge is found after a translation in the functional integral and the integration over $\phi$ :

$$
\begin{aligned}
S & =\frac{1}{2} \int \mathrm{d}^{4} x \sqrt{g}\left(\phi+\frac{Q}{32 \pi} \tilde{\mathrm{G}}_{4} \frac{1}{\Delta_{4}}\right) \Delta_{4}\left(\phi+\frac{Q}{32 \pi} \tilde{\mathrm{G}}_{4} \frac{1}{\Delta_{4}}\right)-\frac{Q^{2}}{(4 \pi)^{2}} \tilde{\mathrm{G}}_{4} \frac{1}{\Delta_{4}} \tilde{\mathrm{G}}_{4} \\
& \rightarrow \frac{1}{2} \operatorname{tr} \ln \left[\sqrt{g} \Delta_{4}\right]-\frac{Q^{2}}{128(4 \pi)^{2}} \tilde{\mathrm{G}}_{4} \frac{1}{\Delta_{4}} \tilde{\mathrm{G}}_{4} .
\end{aligned}
$$

The term proportional to $Q^{2}$ affects the quantity $a$ by the shift written in (2.4). This can be seen using for example the formulas of [20].

No real value of $Q$ can give a positive $a$, as promised. Moreover, the background charge has no effect on $c$. The negative value of $c$ can be checked by computing the stress-tensor two-point function and does not depend on $Q$. The stress tensor reads

$$
\begin{aligned}
T_{\mu \nu}= & -\partial_{\mu} \square \phi \partial_{\nu} \phi-\partial_{\nu} \square \phi \partial_{\mu} \phi-\frac{4}{3} \partial_{\mu} \partial_{\alpha} \phi \partial_{\nu} \partial_{\alpha} \phi+\frac{2}{3} \partial_{\mu} \partial_{\nu} \partial_{\alpha} \phi \partial_{\alpha} \phi+2 \square \phi \partial_{\mu} \partial_{\nu} \phi \\
& +\delta_{\mu \nu}\left[\frac{1}{3} \partial_{\alpha} \square \phi \partial_{\alpha} \phi+\frac{1}{3}\left(\partial_{\alpha} \partial_{\beta} \phi\right)^{2}-\frac{1}{2}(\square \phi)^{2}\right]+\frac{Q}{6 \pi}\left(\partial_{\mu} \partial_{\nu}-\delta_{\mu \nu} \square\right) \square \phi .
\end{aligned}
$$

The two-point function is

$$
\left\langle T_{\mu \nu}(x) T_{\rho \sigma}(0)\right\rangle=-\frac{1}{120 \pi^{4}} \prod_{\mu \nu, \rho \sigma}^{(2)}\left(\frac{1}{|x|^{4}}\right),
$$

in agreement with the value of $c$ given above. We see that the non-unitarity of the theory is exhibited by a violation of reflection positivity. Similarly, the non-unitarity of non-conformal higher-derivative theories, such as a scalar field with lagrangian $\mathcal{L}=\frac{1}{2} \square \phi\left(\square+m^{2}\right) \phi$, is exhibited by poles with negative residues in the propagator [23].

A way to change the signs of both central charges it to consider "higher-derivative anticommuting scalar fields", $\theta, \bar{\theta}$. In this case $Q=0$ and

$$
S=\int \mathrm{d}^{4} x \sqrt{g} \bar{\theta} \Delta_{4} \theta, \quad c=\frac{2}{15}, \quad a=\frac{7}{45} .
$$

This theory can be coupled to, say, the electromagnetic field. In a flat gravitational background the most general renormalizable lagrangian has a finite number of parameters due to the statistics of $\theta$ :

$$
\mathcal{L}=\frac{1}{4} F_{\mu \nu}^{2}+\left|D_{\mu} D_{\mu} \theta\right|^{2}+i F_{\mu \nu} \overline{D_{\mu} \theta} D_{\nu} \theta+\left|D_{\mu} \theta D_{\nu} \theta\right|^{2}+\cdots,
$$

where $D^{2}=D_{\mu} D_{\mu}$. Each term can be further multiplied by a polynomial $1+h \bar{\theta} \theta$. Some simplification comes from the invariance of the theory under the renormalizable change of variables

$$
A_{\mu} \rightarrow A_{\mu}+i \alpha \bar{\theta} \overleftrightarrow{\partial_{\mu}} \theta
$$

$\alpha$ being a parameter of no physical interest. 
The change of the statistics of the fields does not eliminate the non-unitarity of the theory. Indeed, the low-dimensionality of $\theta, \bar{\theta}$ allows us to construct many operators violating the Ferrara-Gatto-Grillo theorem. There are also operators satisfying reflection positivity before the change of statistics and violating it afterwards. For example

$$
\left\langle\left(\partial_{\mu} \bar{\theta} \partial_{\mu} \theta\right)(x)\left(\partial_{\nu} \bar{\theta} \partial_{\nu} \theta\right)(0)\right\rangle=-\left(\partial_{\mu} \partial_{\nu}\langle\bar{\theta}(x) \theta(0)\rangle\right)^{2}<0 .
$$

Finally, there are also operators that have a vanishing two-point function, such as two terms of the electromagnetic current:

$$
j_{\mu}=i\left(\bar{\theta} \square \partial_{\mu} \theta-\partial_{\mu} \square \bar{\theta} \theta+\frac{1}{3} \partial_{\alpha} \bar{\theta} \overleftrightarrow{\partial_{\mu}} \partial_{\alpha} \theta+\frac{4}{3} \square \bar{\theta} \partial_{\mu} \theta-\frac{4}{3} \partial_{\mu} \bar{\theta} \square \theta\right), \quad j_{\mu}^{\prime}=-\frac{i}{2} \pi_{\mu \alpha}\left(\bar{\theta} \overleftrightarrow{\partial_{\alpha}} \theta\right)
$$

We find, defining $J_{\mu}=a j_{\mu}+b j_{\mu}^{\prime}$,

$$
\left\langle j_{\mu}(x) j_{\nu}(0)\right\rangle=\left\langle j_{\mu}^{\prime}(x) j_{\nu}^{\prime}(0)\right\rangle=0, \quad\left\langle J_{\mu}(x) J_{\nu}(0)\right\rangle=-\frac{a b}{4 \pi^{2}} \pi_{\mu \nu}\left(\frac{1}{|x|^{4}}\right) .
$$

Despite the unitarity problem, renormalization of this theory is well-behaved and very presumably there is a conformal window, at least when the gauge field is non-Abelian. Theories such as these are a useful laboratory for the approach of [1].

For fermionic theories

$$
\mathcal{L}=\bar{\psi} \not \partial \square \psi
$$

we have found the stress tensor

$$
\begin{aligned}
T_{\mu \nu}= & h\left\{\bar{\psi}\left(\gamma_{\mu} \partial_{\nu}+\gamma_{\nu} \partial_{\mu}\right) \square \psi-\square \bar{\psi}\left(\gamma_{\mu} \overleftarrow{\partial_{\nu}}+\gamma_{\nu} \overleftarrow{\partial_{\mu}}\right) \psi+3 \square \bar{\psi}\left(\gamma_{\mu} \partial_{\nu}+\gamma_{\nu} \partial_{\mu}\right) \psi\right. \\
& -3 \bar{\psi}\left(\gamma_{\mu} \overleftarrow{\partial_{\nu}}+\gamma_{\nu} \overleftarrow{\partial_{\mu}}\right) \square \psi-\frac{2}{3}\left(\partial_{\mu} \bar{\psi} \overleftrightarrow{\partial} \partial_{\nu} \psi+\partial_{\nu} \bar{\psi} \overleftrightarrow{\partial} \partial_{\mu}\right) \psi+2 \partial_{\alpha} \bar{\psi}\left(\gamma_{\mu} \overleftrightarrow{\partial_{\nu}}+\gamma_{\nu} \overleftrightarrow{\partial_{\mu}}\right) \partial_{\alpha} \psi \\
& -\frac{10}{3}\left(\bar{\psi} \overleftarrow{\partial} \partial_{\mu} \partial_{\nu} \psi-\partial_{\mu} \partial_{\nu} \bar{\psi} \not \partial \psi\right)-\frac{2}{3}\left(\bar{\psi} \not \partial \partial_{\mu} \partial_{\nu} \psi-\partial_{\mu} \partial_{\nu} \bar{\psi} \overleftarrow{\partial} \psi\right) \\
& \left.+\frac{1}{3} \delta_{\mu \nu}\left[7(\bar{\psi} \overleftarrow{\not} \square \psi-\square \bar{\psi} \not \partial \psi)-2 \partial_{\alpha} \bar{\psi} \overleftrightarrow{\not} \partial_{\alpha} \psi\right]\right\},
\end{aligned}
$$

by imposing conservation and tracelessness. It is not straightforward to fix the overall factor $h$ from the coupling to gravity (a Weyl-invariant coupling to external gravity might not exist). The factor could be fixed unambiguously with the OPE technique of sect. 4 or the Noether method, but here we do not need it, since our primary concern is to show that $c$ is negative, independently of the value of $h$. We find

$$
\left\langle T_{\mu \nu}(x) T_{\rho \sigma}(0)\right\rangle=-\frac{8 h^{2}}{15 \pi^{4}} \prod_{\mu \nu, \rho \sigma}^{(2)}\left(\frac{1}{|x|^{4}}\right)<0 .
$$

We might wonder whether the situation changes in higher dimensions, but it is not so. I have checked that a free scalar field with action $\frac{1}{2}(\square \phi)^{2}$ in six dimensions has, again, $c<0$. The 
stress tensor reads

$$
\begin{aligned}
T_{\mu \nu}= & h\left\{\frac{3}{4} \partial_{\mu} \partial_{\alpha} \phi \partial_{\nu} \partial_{\alpha} \phi-\frac{3}{2} \square \phi \partial_{\mu} \partial_{\nu} \phi+\partial_{\nu} \square \phi \partial_{\mu} \phi+\partial_{\mu} \square \phi \partial_{\nu} \phi-\frac{1}{2} \partial_{\mu} \partial_{\nu} \partial_{\alpha} \phi \partial_{\alpha} \phi\right. \\
& \left.-\frac{1}{4} \phi \square \partial_{\mu} \partial_{\nu} \phi+\delta_{\mu \nu}\left[-\frac{1}{4} \partial_{\alpha} \square \phi \partial_{\alpha} \phi-\frac{1}{8}\left(\partial_{\alpha} \partial_{\beta} \phi\right)^{2}+\frac{1}{4}(\square \phi)^{2}\right]\right\}
\end{aligned}
$$

and the two-point function is

$$
\left\langle T_{\mu \nu}(x) T_{\rho \sigma}(0)\right\rangle=-\frac{25 h^{2}}{86016 \pi^{6}} \prod_{\mu \nu, \rho \sigma}^{(2)}\left(\frac{1}{|x|^{6}}\right)<0 .
$$

Summarizing, higher-derivative theories with ordinary statistics have negative central charges, which means that the ghost degrees of freedom prevail over the physical ones. Yet, these theories can be conformal at the classical level and renormalizable at the quantum level. While the unitarity violations can hardly be removed completely, it might be possible that in some conformal theories certain problems are less serious, so that $c$ and $a$ be positive, despite of the underlying non-unitarity. Higher-spin conformal field theories, which I analyse in the rest of the paper, appear to have this property.

\section{Conformal bosonic fields}

The simplest example of higher-spin conformal field theory is the free spin-2 conformal field.

Let $\chi_{\mu \nu}$ be symmetric and traceless. The action

$$
S=\int \mathcal{L}_{1}=\int \frac{1}{2}\left(\partial_{\mu} \chi_{\nu \rho}\right)^{2}-\frac{2}{3}\left(\partial_{\mu} \chi_{\mu \nu}\right)^{2}
$$

is invariant with respect to the coordinate inversion $x^{\mu} \rightarrow \frac{x^{\mu}}{|x|^{2}}$. Under this transformation the tensor $\chi_{\mu \nu}$ transforms as

$$
\chi_{\mu \nu}(x) \rightarrow|x|^{2} I_{\mu \rho}(x) I_{\nu \sigma}(x) \chi_{\rho \sigma}(x),
$$

where $I_{\mu \nu}(x)=\delta_{\mu \nu}-2 x_{\mu} x_{\nu} /|x|^{2}$. This invariance fixes uniquely the action $S$, and the lagrangian $\mathcal{L}_{1}$ up to total derivatives. A better choice of the total derivatives leads to the lagrangian

$$
\mathcal{L}=\frac{1}{2}\left(\partial_{\mu} \chi_{\nu \rho}\right)^{2}-\frac{1}{2} \partial_{\mu} \chi_{\nu \rho} \partial_{\nu} \chi_{\mu \rho}-\frac{1}{6}\left(\partial_{\mu} \chi_{\mu \nu}\right)^{2}
$$

$\mathcal{L}$ transforms as a scalar under coordinate inversion, $\mathcal{L} \rightarrow|x|^{8} \mathcal{L}$. The action $S$ is invariant under the higher-derivative gauge transformation

$$
\delta \chi_{\mu \nu}=\partial_{\mu} \partial_{\nu} \Lambda-\frac{1}{4} \delta_{\mu \nu} \square \Lambda,
$$

but not with respect to the diffeomorphism-type transformation $\delta \chi_{\mu \nu}=\partial_{\mu} \xi_{\nu}+\partial_{\nu} \xi_{\mu}$. The lagrangian $\mathcal{L}$ is also invariant, while $\mathcal{L}_{1}$ is invariant up to a total derivative. 
The gauge transformation (3.8) is compatible with the conformal symmetry. This can be proved by observing that $\Lambda$ has dimension -1 and thus, under coordinate inversion, $\delta \chi_{\mu \nu}$ transforms in the same way as $\chi_{\mu \nu}$ :

$$
\Lambda \rightarrow|x|^{-2} \Lambda, \quad \partial_{\mu} \partial_{\nu} \Lambda-\frac{1}{4} \delta_{\mu \nu} \square \Lambda \rightarrow|x|^{2} I_{\mu \rho}(x) I_{\nu \sigma}(x)\left(\partial_{\rho} \partial_{\sigma} \Lambda-\frac{1}{4} \delta_{\rho \sigma} \square \Lambda\right) .
$$

To check this, observe that the derivative operator transforms as a vector of dimension 1: $\partial_{\mu} \rightarrow|x|^{2} I_{\mu \nu}(x) \partial_{\nu}$.

The field equations read

$$
\square \chi_{\mu \nu}=\frac{2}{3} \partial_{\rho}\left(\partial_{\mu} \chi_{\nu \rho}+\partial_{\nu} \chi_{\mu \rho}\right)-\frac{1}{3} \delta_{\mu \nu} \partial_{\rho} \partial_{\sigma} \chi_{\rho \sigma} .
$$

Defining the vector field $\mathcal{A}_{\mu}=\partial_{\nu} \chi_{\mu \nu}$ and its field strength $\mathcal{F}_{\mu \nu}=\partial_{\mu} \mathcal{A}_{\nu}-\partial_{\nu} \mathcal{A}_{\mu}$, the field equations and gauge invariance imply

$$
\partial_{\mu} \mathcal{F}_{\mu \nu}=0, \quad \delta \mathcal{A}_{\mu}=\frac{3}{4} \partial_{\mu} \square \Lambda .
$$

\subsection{Field strength}

The gauge symmetry (3.8) leads to the introduction of a natural field strength,

$$
F_{\mu \nu \alpha}=\partial_{\mu} \chi_{\nu \alpha}-\partial_{\nu} \chi_{\mu \alpha}-\frac{1}{3} \delta_{\mu \alpha} \partial_{\rho} \chi_{\rho \nu}+\frac{1}{3} \delta_{\nu \alpha} \partial_{\rho} \chi_{\rho \mu},
$$

which is easily proved to be gauge-invariant. This field strength satisfies a number of noticeable properties. First of all, we have the identities

$$
F_{\mu \nu \alpha}=-F_{\nu \mu \alpha}, \quad F_{\mu \nu \mu}=0, \quad F_{\mu \nu \alpha}+F_{\alpha \mu \nu}+F_{\nu \alpha \mu}=0 .
$$

The third identity will be called the cyclic identity. Secondly, the lagrangian (3.7) can be written as

$$
\mathcal{L}=\frac{1}{4}\left(F_{\mu \nu \alpha}\right)^{2}
$$

which implies, in particular, that it is positive-definite, a fact that was not evident from (3.6) and (3.7). Since $\mathcal{L}$ is a conformal field of dimension 4, it is evident that the field strength is itself a conformal field of dimension 2 and transforms as

$$
F_{\mu \nu \alpha} \rightarrow|x|^{4} I_{\mu \rho}(x) I_{\nu \sigma}(x) I_{\alpha \beta}(x) F_{\rho \sigma \beta}
$$

under coordinate inversion. The field equations read

$$
\partial_{\mu} F_{\mu \nu \alpha}+\partial_{\mu} F_{\mu \alpha \nu}=0 .
$$

It is convenient to introduce the dual of the field strength, as well as self-dual and anti-selfdual field strengths:

$$
\tilde{F}_{\mu \nu \alpha}=\frac{1}{2} \varepsilon_{\mu \nu \rho \sigma} F_{\rho \sigma \alpha}, \quad F_{\mu \nu \alpha}^{ \pm}=\frac{1}{2}\left(F_{\mu \nu \alpha} \pm \tilde{F}_{\mu \nu \alpha}\right) .
$$


Each of these tensors satisfies the same symmetry identities (3.9) as $F_{\mu \nu \alpha}$. We can also derive the "Bianchi identity"

$$
\partial_{\mu} \tilde{F}_{\mu \nu \alpha}+\partial_{\mu} \tilde{F}_{\mu \alpha \nu}=0 .
$$

There is a natural topological invariant and a "Chern-Simons" form:

$$
F_{\mu \nu \alpha} \tilde{F}_{\mu \nu \alpha}=\partial_{\mu}\left(\varepsilon_{\mu \nu \rho \sigma} \chi_{\nu \alpha} F_{\rho \sigma \alpha}\right) .
$$

Non-trivial interactions for conformal higher-spin theories can be constructed, as power series in the field strength:

$$
\mathcal{L}=\frac{1}{4}\left(F_{\mu \nu \alpha}\right)^{2}+\frac{1}{\Lambda^{4}}\left\{a\left[\left(F_{\mu \nu \alpha}\right)^{2}\right]^{2}+b F_{\mu \nu \alpha} F_{\nu \rho \alpha} F_{\rho \sigma \beta} F_{\sigma \mu \beta}+c F_{\mu \nu \alpha} F_{\nu \rho \beta} F_{\rho \sigma \alpha} F_{\sigma \mu \beta}\right\}+\cdots
$$

$\Lambda$ being some mass scale and $a, b, c$ being dimensionless parameters. These vertices are nontrivial because they do not vanish when the field equations (3.10) are satisfied. Our interest, however, is mostly to look for non-trivial renormalizable interactions, which preserve conformality at the classical level. These are more difficult to construct, but are fundamental for the conformal hypothesis stated in the introduction. Certain renormalizable interactions will be studied in this paper (for fermionic higher-spin conformal theories), but a complete classification will not be given here.

The coupling to gravity is not straightforward and might not exist at all. Simple attempts to impose the compatibility between the gauge symmetry (3.8) and gravity generate terms that cannot be reabsorbed. Nevertheless, this does not forbid a correct definition of $c$ and $a$ (see section 3$)$.

\subsection{Propagator and ghosts}

In this section I calculate the propagators and discuss a number of important features. The quantization is performed in the BRS approach and in the framework of the functional integral.

The most natural gauge-fixing is

$$
\partial_{\mu} \partial_{\nu} \chi_{\mu \nu}=\partial_{\mu} \mathcal{A}_{\mu}=0 .
$$

The gauge-fixed lagrangian becomes

$$
\mathcal{L}_{1}=\frac{1}{2}\left(\partial_{\mu} \chi_{\nu \rho}\right)^{2}-\frac{2}{3}\left(\partial_{\mu} \chi_{\mu \nu}\right)^{2}+b \partial_{\mu} \partial_{\nu} \chi_{\mu \nu}-\frac{3}{4} \bar{C} \square^{2} C
$$

and the BRS transformation reads

$$
s \chi_{\mu \nu}=\partial_{\mu} \partial_{\nu} C-\frac{1}{4} \delta_{\mu \nu} \square C, \quad s C=0, \quad s \bar{C}=b, \quad s b=0 .
$$

Defining the projectors

$$
\begin{aligned}
P_{1 \mu \nu, \rho \sigma} & =\frac{1}{2}\left(\delta_{\mu \rho} \delta_{\nu \sigma}+\delta_{\mu \sigma} \delta_{\nu \rho}\right)-\frac{1}{4} \delta_{\mu \nu} \delta_{\rho \sigma}, \\
P_{2 \mu \nu, \rho \sigma} & =\frac{1}{3}\left(\partial_{\mu} \partial_{\rho} \delta_{\nu \sigma}+\partial_{\mu} \partial_{\sigma} \delta_{\nu \rho}+\partial_{\nu} \partial_{\rho} \delta_{\mu \sigma}+\partial_{\nu} \partial_{\sigma} \delta_{\mu \rho}-\partial_{\mu} \partial_{\nu} \delta_{\rho \sigma}-\partial_{\rho} \partial_{\sigma} \delta_{\mu \nu}+\frac{1}{4} \square \delta_{\mu \nu} \delta_{\rho \sigma}\right) \frac{1}{\square}, \\
\varpi_{\mu \nu} & =\partial_{\mu} \partial_{\nu} \frac{1}{\square}-\frac{1}{4} \delta_{\mu \nu},
\end{aligned}
$$


we have the relations

$$
P_{1}^{2}=P_{1}, \quad P_{1} P_{2}=P_{2}, \quad P_{2}^{2}=\frac{2}{3} P_{2}+\frac{4}{9} \varpi \varpi, \quad P_{1} \varpi=\varpi, \quad P_{2} \varpi=\varpi, \quad \operatorname{tr} \varpi \varpi=\frac{3}{4} .
$$

The lagrangian, written as

$$
\mathcal{L}_{1}+\frac{3}{4} \bar{C} \square^{2} C=-\frac{1}{2}(\chi b) Q\left(\begin{array}{l}
\chi \\
b
\end{array}\right)=-\frac{1}{2}(\chi b) \square\left[\begin{array}{cc}
P_{1}-P_{2} & -\varpi \\
-\varpi & 0
\end{array}\right]\left(\begin{array}{l}
\chi \\
b
\end{array}\right),
$$

can be easily inverted to find the propagators, which are

$$
\left\langle\left(\begin{array}{l}
\chi \\
b
\end{array}\right)(\chi b)\right\rangle=-\left[\begin{array}{cc}
P_{1}+3 P_{2}-\frac{16}{3} \varpi \varpi & -\frac{4}{3} \varpi \\
-\frac{4}{3} \varpi & 0
\end{array}\right] \frac{1}{\square}
$$

The $x$-space propagators can be written using

$$
-\left(\frac{1}{\square}\right)_{(x, 0)}=\frac{1}{4 \pi^{2}} \frac{1}{|x|^{2}}, \quad \frac{1}{\square^{2}}=-\frac{1}{16 \pi^{2}} \ln |x|^{2} \mu^{2}, \quad \frac{1}{\square^{3}}=-\frac{|x|^{2}}{128 \pi^{2}}\left(\ln |x|^{2} \mu^{2}-\frac{3}{2}\right) .
$$

The field $b$ does not propagate, because $\langle b(x) b(0)\rangle=\langle s(\bar{C}(x) b(0))\rangle=0$. Similarly, $\left\langle b(x) \chi_{\mu \nu}(0)\right\rangle$ vanishes on-shell, i.e. when saturated by $\chi$-polarizations satisfying the gauge-fixing condition $\partial_{\mu} \partial_{\nu} \chi_{\mu \nu}=0$.

The ghosts of the theory are the components $\mathcal{A}_{\mu}$ and a way to single out the (two) physical degrees of freedom is to set

$$
\mathcal{A}_{\mu}=\partial^{\mu} \chi_{\mu \nu}=0 \text {. }
$$

This condition has no dynamical origin (the theory has $\mathcal{A}_{\mu} \neq 0$ ) and is here meant for a pedagogical purpose. Observe that only when $\mathcal{A}_{\mu}=0$ the field equations reduce to an ordinary wave equation for $\chi_{\mu \nu}, \square \chi_{\mu \nu}=0$. Moreover, the propagator, saturated with $\chi$-polarizations, becomes $\left|\chi_{\mu \nu}(k)\right|^{2} / k^{2}$ in this case. The gauge-fixing condition $\partial_{\mu} \partial_{\nu} \chi_{\mu \nu}=0$ gives, in momentum space,

$$
\chi_{00}+\hat{n}_{i} \hat{n}_{j} \chi_{i j}=2 \hat{n}_{i} \chi_{0 i},
$$

where $\hat{n}_{i}=k_{i} / k_{0}, i=1,2,3$, and $k_{0}^{2}=k_{i}^{2}$. When $\mathcal{A}_{\mu}=0$, the additional conditions $\partial_{\mu} \chi_{\mu i}=0$ give

$$
\hat{n}_{j} \chi_{i j}=\chi_{0 i}
$$

which, reinserted into (3.14), also give

$$
\chi_{00}=\hat{n}_{i} \chi_{0 i}
$$

i.e. $\partial_{\mu} \chi_{\mu 0}=0$, justifying (3.13). The condition of vanishing trace for $\chi_{\mu \nu}$ gives $\chi_{i i}=-\chi_{00}=$ $\hat{n}_{i} \hat{n}_{j} \chi_{i j}$. We have therefore

$$
\left|\chi_{\mu \nu}\right|^{2}=\left|\chi_{i j}\right|^{2}+\left|\chi_{i i}\right|^{2}-2\left|\hat{n}_{j} \chi_{i j}\right|^{2} .
$$


Let us choose $\hat{n}_{i}=(0,0,1)$. The condition $\chi_{i i}=\hat{n}_{i} \hat{n}_{j} \chi_{i j}$ gives $\chi_{22}=-\chi_{11}$ and finally

$$
\left|\chi_{\mu \nu}\right|^{2}=2\left(\left|\chi_{11}\right|^{2}+\left|\chi_{12}\right|^{2}\right) \geq 0
$$

Concluding, the two physical degrees of freedom are $\chi_{11}$ and $\chi_{12}$, the unphysical degrees of freedom are $\mathcal{A}_{\mu}$. The question is which of the two prevail in $c$ and $a$. If the central charges are positive, the physical degrees of freedom prevail over the unphysical ones. We compute $c$ and $a$ in section 4 .

\subsection{Arbitrary integer spin}

Let $\chi_{\mu_{1} \cdots \mu_{s}}$ be a completely symmetric and completely traceless tensor. Invariance of the action under the transformation

$$
\chi_{\mu_{1} \cdots \mu_{s}} \rightarrow|x|^{2} I_{\mu_{1}}^{\nu_{1}}(x) \cdots I_{\mu_{s}}^{\nu_{s}}(x) \chi_{\nu_{1} \cdots \nu_{s}}
$$

fixes uniquely the lagrangian

$$
\mathcal{L}_{1}=\frac{1}{2}\left(\partial_{\alpha} \chi_{\mu_{1} \cdots \mu_{s}}\right)^{2}-\frac{s}{s+1}\left(\partial_{\alpha} \chi_{\alpha \mu_{2} \cdots \mu_{s}}\right)^{2},
$$

up to the overall factor and total derivatives. $\mathcal{L}_{1}$ reduces to the usual vector lagrangian for $s=1$ and to the free real-scalar theory for $s=0$. The action is invariant under the gauge transformation

$$
\delta \chi_{\mu_{1} \cdots \mu_{s}}=\partial_{\mu_{1}} \cdots \partial_{\mu_{s}} \Lambda-\text { traces, }
$$

which, as before, is compatible with (3.15), when taking into account that $\Lambda$ has dimension $1-s$ and transforms as $\Lambda \rightarrow|x|^{2(1-s)} \Lambda$ under coordinate inversion.

The field strength reads

$$
F_{\mu \nu \mu_{2} \cdots \mu_{s}}=\partial_{\mu} \chi_{\nu \mu_{2} \cdots \mu_{s}}-\partial_{\nu} \chi_{\mu \mu_{2} \cdots \mu_{s}}-\frac{1}{s+1} \sum_{i=2}^{s}\left(\delta_{\mu \mu_{i}} \partial_{\alpha} \chi_{\alpha \nu \mu_{2} \cdots \widehat{\mu_{i} \cdots \mu_{s}}}-\delta_{\nu \mu_{i}} \partial_{\alpha} \chi_{\alpha \mu \mu_{2} \cdots \widehat{\mu_{i} \cdots \mu_{s}}}\right) \text {. }
$$

A hat denotes indices that have to be omitted. As before, the field strength is gauge-invariant and conformal. It is completely symmetric in $\mu_{2} \cdots \mu_{s}$ and antisymmetric in $\mu \nu$. Furthermore, it is completely traceless, not only in the indices $\mu_{2} \cdots \mu_{s}$, but also with respect to the remaining contraction:

$$
F_{\mu \nu \nu \mu_{3} \cdots \mu_{s}}=0
$$

Finally, it satisfies the cyclic condition

$$
F_{\mu \nu \alpha \mu_{3} \cdots \mu_{s}}+F_{\alpha \mu \nu \mu_{3} \cdots \mu_{s}}+F_{\nu \alpha \mu \mu_{3} \cdots \mu_{s}}=0 .
$$

The conformal, positive-definite lagrangian can be written as

$$
\mathcal{L}=\frac{1}{4}\left(F_{\mu \nu \mu_{2} \cdots \mu_{s}}\right)^{2}
$$


The field equations and Bianchi identities are

$$
\partial_{\mu} F_{\mu \alpha_{1} \cdots \alpha_{s}}+\operatorname{perms}\left(\alpha_{1} \cdots \alpha_{s}\right)=0, \quad \partial_{\mu} \tilde{F}_{\mu \alpha_{1} \cdots \alpha_{s}}+\operatorname{perms}\left(\alpha_{1} \cdots \alpha_{s}\right)=0 .
$$

Dual and self-dual field strengths are defined as

$$
\tilde{F}_{\mu \nu \alpha_{2} \cdots \alpha_{s}}=\frac{1}{2} \varepsilon_{\mu \nu \rho \sigma} F_{\rho \sigma \alpha_{2} \cdots \alpha_{s}}, \quad F_{\mu \nu \alpha_{2} \cdots \alpha_{s}}^{ \pm}=\frac{1}{2}\left(F_{\mu \nu \alpha_{2} \cdots \alpha_{s}} \pm \tilde{F}_{\mu \nu \alpha_{2} \cdots \alpha_{s}}\right)
$$

and satisfy the traceless and cyclic conditions (3.16) and (3.17).

There is a topological invariant, proportional to the integral of

$$
F_{\mu \nu \mu_{2} \cdots \mu_{s}} \tilde{F}_{\mu \nu \mu_{2} \cdots \mu_{s}}=\partial_{\mu}\left(\varepsilon_{\mu \nu \rho \sigma} \chi_{\nu \mu_{2} \cdots \mu_{s}} F_{\rho \sigma \mu_{2} \cdots \mu_{s}}\right) .
$$

The equality can be proved by using the Bianchi identity and (3.16).

The stress tensor (see the discussion of sect. 4.1) is

$$
T_{\mu \nu}=\text { const. } F_{\mu \alpha_{1} \cdots \alpha_{s}}^{+} F_{\nu \alpha_{1} \cdots \alpha_{s}}^{-} .
$$

Tracelessness is straightforward, while the proof of conservation follows the same line as in the spin-2 case. The procedure to fix the overall factor and the comparison with the Noether tensor are discussed in detail for $s=2$. Higher-spin tensor currents can be constructed using the recipes of [26, 27].

\subsection{Implications of the higher-derivative gauge invariance on correlators}

The general form of the two-point function of a conformal composite operator $\mathcal{O}_{\mu_{1} \cdots \mu_{s}}$ with spin $s$ is, in the notation of [26, 27]:

$$
\left\langle\mathcal{O}_{\mu_{1} \cdots \mu_{s}}(x) \mathcal{O}_{\nu_{1} \cdots \nu_{s}}(0)\right\rangle=c_{s} \frac{1}{(|x| \mu)^{2 h_{s}}} \prod_{\mu_{1} \cdots \mu_{s}, \nu_{1} \cdots \nu_{s}}^{(s)}\left(\frac{1}{|x|^{4}}\right),
$$

where $\prod_{\mu_{1} \cdots \mu_{s}, \nu_{1} \cdots \nu_{s}}^{(s)}$ is the unique differential operator of degree $2 s$ that is completely symmetric and traceless in $\mu_{1} \cdots \mu_{s}$ and $\nu_{1} \cdots \nu_{s}$, symmetric in the exchange $\mu \leftrightarrow \nu$, conserved with respect to any index. For example, $\pi_{\mu \nu}=\partial_{\mu} \partial_{\nu}-\delta_{\mu \nu} \square$ for $s=1$, while $\prod_{\mu \nu, \rho \sigma}^{(2)}=\frac{1}{2}\left(\pi_{\mu \rho} \pi_{\nu \sigma}+\pi_{\mu \sigma} \pi_{\nu \rho}\right)-$ $\frac{1}{3} \pi_{\mu \nu} \pi_{\rho \sigma}$. The factor $c_{s}$ is a constant (higher-spin central charge) and $h_{s}$ is equal to $\delta_{s}-s-2$, where $\delta_{s}$ is the total dimension of the operator $\mathcal{O}_{\mu_{1} \cdots \mu_{s}}$.

If the operator $\mathcal{O}_{\mu_{1} \cdots \mu_{s}}$ couples to a conformal higher-spin field $\chi_{\mu_{1} \cdots \mu_{s}}$, via a vertex $\mathcal{O}_{\mu_{1} \cdots \mu_{s}} \chi_{\mu_{1} \cdots \mu_{s}}$, then the following "multiple-conservation" condition holds:

$$
\partial_{\mu_{1}} \cdots \partial_{\mu_{s}} \mathcal{O}_{\mu_{1} \cdots \mu_{s}}=0
$$

An ordinary conservation condition $\partial_{\mu_{s}} \mathcal{O}_{\mu_{1} \cdots \mu_{s}}=0$ implies $h_{s}=0$. Instead, applying the multiple-conservation condition (3.19) to the correlator (3.18), we find that $h_{s}$ can take an arbitrary integer value between 0 and $1-s$. Consequently, we have the following spectrum of allowed dimensions:

$$
\delta_{s}=2+s, 1+s, \cdots, 3
$$


Observe that only the operators of dimension 3 need $s$ divergences to be annihilated. Operators of higher dimension are allowed to satisfy more restrictive conditions. In particular, operators of dimension $2+s$ can be conserved in the usual sense $\left(\partial_{\mu_{1}} \mathcal{O}_{\mu_{1} \cdots \mu_{s}}=0\right)$, operators of dimension $1+s$ can be annihilated by two divergences $\left(\partial_{\mu_{1}} \partial_{\mu_{2}} \mathcal{O}_{\mu_{1} \cdots \mu_{s}}=0\right)$, etc.

The Ferrara-Gatto-Grillo theorem [9] says that in a unitary theory primary conformal operators with spin- $s$ have dimensions $\delta_{s} \geq 2+s$. This property is here violated. We see from (3.20) that the minimal allowed dimension is 3 . This feature is relevant to the conformal hypothesis stated in the introduction: the interaction vertex

$$
\mathcal{O}_{\mu_{1} \cdots \mu_{s}} \chi_{\mu_{1} \cdots \mu_{s}}
$$

is renormalizable if $\mathcal{O}_{\mu_{1} \cdots \mu_{s}}$ is such an operator of dimension 3 ; therefore, in our theories, renormalizable higher-spin interactions are not ruled out in a trivial way (see section 5.1).

\section{The spin-2 conformal boson in detail}

In this section I study the stress tensor of the spin-2 conformal boson, compute its two-point function and $\mathrm{OPE}$, and extract the values of the central charges $c$ and $a$. The result is that both $c$ and $a$ have positive values.

\subsection{Stress-tensor, spin-2 currents and the definitions of $c$ and $a$}

The Noether method produces a non-gauge-invariant, non-symmetric, traceful stress tensor

$$
T_{\mu \nu}^{\mathrm{N}}=\partial_{\mu} \chi_{\alpha \beta} F_{\nu \alpha \beta}-\frac{1}{4} \delta_{\mu \nu} F_{\alpha \beta \gamma}^{2} .
$$

This operator is conserved in $\nu\left(\partial_{\nu} T_{\mu \nu}=0\right)$, gauge-invariant and traceless up to total derivatives, and it does not transform simply under coordinate inversion $x_{\mu} \rightarrow x_{\mu} /|x|^{2}$. For this reason, it is not easy to use the Noether tensor to extract $c$ and $a$. Moreover, no improvement term $\psi_{\mu \nu \lambda}=-\psi_{\mu \lambda \nu}$ appears to be such that $T_{\mu \nu}^{\mathrm{N}}+\partial_{\lambda} \psi_{\mu \nu \lambda}$ transform correctly and be gauge-invariant.

There exists, nevertheless, a remarkable spin-2 tensor:

$$
T_{\mu \nu}=\frac{8}{3} F_{\mu \alpha \beta}^{+} F_{\nu \alpha \beta}^{-}=\frac{4}{3} F_{\mu \alpha \beta} F_{\nu \alpha \beta}-\frac{1}{3} \delta_{\mu \nu} F_{\alpha \beta \gamma}^{2},
$$

which appears to have the desired properties. We are going to show that this tensor gives sensible definitions of $c$ and $a$ and compute their values.

The unusual factor will be fixed in the next section by checking the Poincaré algebra in the operator-product expansion.

It is straightforward to show that $T_{\mu \nu}$ is traceless, gauge-invariant and conserved when the field equations (3.10) are satisfied, and transforms correctly under coordinate inversion. For the proof of conservation we observe that the cyclic identity also implies

$$
T_{\mu \nu}=\frac{8}{3} F_{\mu \alpha \beta}^{+} F_{\nu \beta \alpha}^{-}
$$


The difference $\Delta$ between the two forms for $T_{\mu \nu}$ is proportional to $F_{\mu \alpha \beta}^{+} F_{\nu[\beta \alpha]}^{-}$, the brackets denoting antisymmetrization. The cyclic identity in (3.9) can be expressed as $F_{\mu \nu \alpha}-F_{\mu \alpha \nu}=$ $F_{\alpha \nu \mu}$. Similar expressions hold for $\tilde{F}$ and $F^{ \pm}$. We have therefore $\Delta \sim F_{\mu \alpha \beta}^{+} F_{\alpha \beta \nu}^{-}$. Using the cyclic identity once more on $F^{+}$we arrive at $\Delta \propto F_{\alpha \beta \mu}^{+} F_{\alpha \beta \nu}^{-}=0$.

We are going, with some abuse of language, to call "stress tensor" the spin-2 current (4.21), because this helps us use formulas from the literature. It is clear, on the other hand, that it is a fairly new object and demands a special study. At the moment, however, I cannot characterize it any better, and the properties outlined here are meant to draw attention to it.

\subsection{Computation of $c$}

The field-strength propagator $\left\langle F_{\mu \nu \alpha}(x) F_{\rho \sigma \beta}(0)\right\rangle$ is, by conformal invariance, $1 /|x|^{2 d}$ times a linear combination of the following three conformal structures:

$$
\begin{aligned}
C_{\mu \nu \alpha, \rho \sigma \beta}^{(1)}(x) & =\left(I_{\mu \rho}(x) I_{\nu \sigma}(x)-I_{\mu \sigma}(x) I_{\nu \rho}(x)\right) I_{\alpha \beta}(x), \\
C_{\mu \nu \alpha, \rho \sigma \beta}^{(2)} & =\left(I_{\mu \beta} I_{\nu \rho}-I_{\nu \beta} I_{\mu \rho}\right) I_{\sigma \alpha}-(\rho \leftrightarrow \sigma), \\
C_{\mu \nu \alpha, \rho \sigma \beta}^{(3)} & =\left(\delta_{\mu \alpha} I_{\nu \rho}-\delta_{\nu \alpha} I_{\mu \rho}\right) \delta_{\sigma \beta}-(\rho \leftrightarrow \sigma),
\end{aligned}
$$

where $d$ is the dimension of $F$ (2 in the free-field limit). The trace and cyclic conditions (3.9) fix the combination of $C^{(1)}, C^{(2)}$ and $C^{(3)}$ uniquely up to the overall factor, which can be found by direct inspection, using the $\chi$-propagator worked out in the previous section. The final result reads

$$
\left\langle F_{\mu \nu \alpha}(x) F_{\rho \sigma \beta}(0)\right\rangle=\frac{1}{2 \pi^{2}} \frac{1}{|x|^{4}}\left(2 C_{\mu \nu \alpha, \rho \sigma \beta}^{(1)}-C_{\mu \nu \alpha, \rho \sigma \beta}^{(2)}+C_{\mu \nu \alpha, \rho \sigma \beta}^{(3)}\right) .
$$

A good check is that this correlator satisfies the field equations (3.10).

With (4.22) we find the two-point function:

$$
\left\langle T_{\mu \nu}(x) T_{\rho \sigma}(0)\right\rangle=\frac{4}{45 \pi^{4}} \prod_{\mu \nu, \rho \sigma}^{(2)}\left(\frac{1}{|x|^{4}}\right),
$$

and this defines the central charge $c$. We have

$$
c=\frac{32}{45}
$$

\subsection{OPE structure and computation of $a$}

The OPE structure exhibits novel features with respect to the ordinary theories. In particular, the presence of ghosts is exhibited by higher-spin composite operators of low dimensionality. The OPE of two stress tensors contains: the central charge $c$, with singularity $1 /|x|^{8}$; the stress tensor itself, with singularity $1 /|x|^{4}$; higher-spin currents of dimension $2+s, 1+s, \cdots 3$, where $s$ is the spin; descendants and regular terms. The first higher-spin current is a spin- 4 , dimension- 4 operator appearing at the same level as the stress tensor (singularity $1 /|x|^{4}$ ). This operator 
reads

$$
\mathcal{O}_{\mu \nu \rho \sigma}^{(4)}=\sum_{\operatorname{perms}(\mu \nu \rho \sigma)}^{\prime} F_{\alpha \mu \nu}^{+} F_{\alpha \rho \sigma}^{-}-\text {traces. }
$$

The primed sum is understood to be divided by the number of permutations. The operator $\mathcal{O}_{\mu \nu \rho \sigma}^{(4)}$ satisfies the multiple-conservation condition $\partial_{\mu} \partial_{\nu} \partial_{\rho} \partial_{\sigma} \mathcal{O}_{\mu \nu \rho \sigma}^{(4)}=0$. The proof of this fact is lengthy and involves repeated use of the cyclic identity and the field equations. Observe in particular that $\partial_{\mu} \partial_{\nu} F_{\alpha \mu \nu}=0$ on the solutions to the field equations. I illustrate the strategy of the proof on the most involved term, which is

$$
\partial_{\rho} \partial_{\sigma} F_{\alpha \mu \nu}^{+} \partial_{\mu} \partial_{\nu} F_{\alpha \rho \sigma}^{-}
$$

First, we exchange $\mu$ and $\rho$ by using the property of self-duality in $\alpha \mu$ and anti-self-duality in $\alpha \rho$. We then use the cyclic identity on $F_{\alpha \mu \sigma}^{-}$and arrive at

$$
-\partial_{\rho} \partial_{\sigma} F_{\alpha \rho \nu}^{+} \partial_{\mu} \partial_{\nu}\left(F_{\mu \sigma \alpha}^{-}+F_{\sigma \alpha \mu}^{-}\right) .
$$

We use the field equations to replace $\partial_{\mu} F_{\mu \sigma \alpha}^{-}$with $\partial_{\mu} F_{\alpha \mu \sigma}^{-}$and observe that we obtain a term identical to the one we started from. We move it on the left-hand side and write

$$
\partial_{\rho} \partial_{\sigma} F_{\alpha \mu \nu}^{+} \partial_{\mu} \partial_{\nu} F_{\alpha \rho \sigma}^{-}=\frac{1}{2} \partial_{\rho} \partial_{\sigma} F_{\alpha \rho \nu}^{+} \partial_{\mu} \partial_{\nu} F_{\alpha \sigma \mu}^{-} \text {. }
$$

Now we use the cyclic identity on $F_{\alpha \rho \nu}^{+}$and get

$$
-\frac{1}{2} \partial_{\rho} \partial_{\sigma}\left(F_{\rho \nu \alpha}^{+}+F_{\nu \alpha \rho}^{+}\right) \partial_{\mu} \partial_{\nu} F_{\alpha \sigma \mu}^{-}
$$

Using the field equation $\partial_{\rho} F_{\rho \nu \alpha}^{+}=\partial_{\rho} F_{\alpha \rho \nu}^{+}$we finally arrive at

$$
-\frac{1}{4} \partial_{\rho} \partial_{\sigma} F_{\nu \alpha \rho}^{+} \partial_{\mu} \partial_{\nu} F_{\alpha \nu \mu}^{-}=-\frac{1}{4} \partial_{\rho} \partial_{\sigma} F_{\sigma \alpha \rho}^{+} \partial_{\mu} \partial_{\nu} F_{\alpha \sigma \mu}^{-}=0 .
$$

The other non-trace terms in $\mathcal{O}_{\mu \nu \rho \sigma}^{(4)}$ can be shown to vanish in a similar way. Finally, the trace terms always contain the stress tensor and obey the multiple-conservation condition because the stress tensor is conserved.

In the basis of [26] we find the OPE expansion

$$
\begin{aligned}
& T_{\mu \nu}(x) T_{\rho \sigma}(0)= \frac{4}{45 \pi^{4}} \prod_{\mu \nu, \rho \sigma}^{(2)}\left(\frac{1}{|x|^{4}}\right) \\
&+\frac{1}{4 \pi^{2}} T_{\alpha \beta}(0) \quad\left[\operatorname{SP}_{\mu \nu, \rho \sigma ; \alpha \beta}\left(\frac{1}{|x|^{2}}\right)+\frac{3}{32} \prod_{\mu \nu, \rho \sigma}^{(2)} \partial_{\alpha} \partial_{\beta}\left(|x|^{2} \ln |x|^{2} \mu^{2}\right)\right. \\
&\left.\quad-\frac{5}{32} \prod_{\mu \nu \alpha, \beta \rho \sigma}^{(3)}\left(|x|^{2} \ln |x|^{2} \mu^{2}\right)\right] \\
&+\frac{1}{4 \pi^{2}} \mathcal{O}_{\alpha \beta \gamma \delta}^{(4)}(0) \quad\left[-\frac{1}{45} \prod_{\mu \nu, \rho \sigma}^{(2)} \partial_{\alpha} \partial_{\beta} \partial_{\gamma} \partial_{\delta}\left(|x|^{4} \ln |x|^{2} \mu^{2}\right)\right. \\
&\left.+\frac{5}{126} \prod_{\mu \nu \alpha, \rho \sigma \beta}^{(3)} \partial_{\gamma} \partial_{\delta}\left(|x|^{4} \ln |x|^{2} \mu^{2}\right)-\frac{1}{216} \prod_{\mu \nu \rho \sigma, \alpha \beta \gamma \delta}^{(4)}\left(|x|^{4} \ln |x|^{2} \mu^{2}\right)\right] \\
&+ \text { less singular terms }+ \text { descendants }+ \text { regular terms, }
\end{aligned}
$$


the structure $\mathrm{SP}_{\mu \nu, \rho \sigma ; \alpha \beta}\left(\frac{1}{|x|^{2}}\right)$ being the generator of the Poincaré algebra. The overall coefficient of $T_{\mu \nu}$ has been fixed by matching the coefficient of $T_{\alpha \beta} \mathrm{SP}_{\mu \nu, \rho \sigma ; \alpha \beta}\left(\frac{1}{|x|^{2}}\right)$ in the OPE, which is universal and has to be equal to $1 / 4 \pi^{2}$.

In the calculation of the above OPE, it is necessary to extract the spin- 2 content from the product $F_{\mu \nu \alpha}^{+} F_{\rho \sigma \beta}^{-}+F_{\mu \nu \alpha}^{-} F_{\rho \sigma \beta}^{+}$. It can be proved that the stress-tensor content of this expression is fixed uniquely by the symmetry properties in the indices, the cyclic identity, the tracelessness of $F$, and relations such as $F_{\alpha \beta \nu}^{+} F_{\alpha \beta \sigma}^{-}=0, F_{\mu \alpha \beta}^{+} F_{\nu \alpha \beta}^{-}=\frac{3}{8} T_{\mu \nu}$, with the result

$$
\begin{array}{r}
F_{\mu \nu \alpha}^{+} F_{\rho \sigma \beta}^{-}+F_{\mu \nu \alpha}^{-} F_{\rho \sigma \beta}^{+} \rightarrow \frac{3}{128}\left(-2 \delta_{\mu \sigma} \delta_{\nu \rho} T_{\alpha \beta}+2 \delta_{\mu \rho} \delta_{\nu \sigma} T_{\alpha \beta}+3 \delta_{\beta \sigma} \delta_{\nu \rho} T_{\alpha \mu}-3 \delta_{\beta \rho} \delta_{\nu \sigma} T_{\alpha \mu}\right. \\
-3 \delta_{\beta \sigma} \delta_{\mu \rho} T_{\alpha \nu}+3 \delta_{\beta \rho} \delta_{\mu \sigma} T_{\alpha \nu}-\delta_{\beta \nu} \delta_{\mu \sigma} T_{\alpha \rho}+\delta_{\beta \mu} \delta_{\nu \sigma} T_{\alpha \rho}+\delta_{\beta \nu} \delta_{\mu \rho} T_{\alpha \sigma}-\delta_{\beta \mu} \delta_{\nu \rho} T_{\alpha \sigma}-\delta_{\alpha \sigma} \delta_{\nu \rho} T_{\beta \mu} \\
+\delta_{\alpha \rho} \delta_{\nu \sigma} T_{\beta \mu}+\delta_{\alpha \sigma} \delta_{\mu \rho} T_{\beta \nu}-\delta_{\alpha \rho} \delta_{\mu \sigma} T_{\beta \nu}+3 \delta_{\alpha \nu} \delta_{\mu \sigma} T_{\beta \rho}-3 \delta_{\alpha \mu} \delta_{\nu \sigma} T_{\beta \rho}-3 \delta_{\alpha \nu} \delta_{\mu \rho} T_{\beta \sigma}+3 \delta_{\alpha \mu} \delta_{\nu \rho} T_{\beta \sigma} \\
+4 \delta_{\alpha \sigma} \delta_{\beta \nu} T_{\mu \rho}-4 \delta_{\alpha \nu} \delta_{\beta \sigma} T_{\mu \rho}+5 \delta_{\alpha \beta} \delta_{\nu \sigma} T_{\mu \rho}-4 \delta_{\alpha \rho} \delta_{\beta \nu} T_{\mu \sigma}+4 \delta_{\alpha \nu} \delta_{\beta \rho} T_{\mu \sigma}-5 \delta_{\alpha \beta} \delta_{\nu \rho} T_{\mu \sigma} \\
\left.-4 \delta_{\alpha \sigma} \delta_{\beta \mu} T_{\nu \rho}+4 \delta_{\alpha \mu} \delta_{\beta \sigma} T_{\nu \rho}-5 \delta_{\alpha \beta} \delta_{\mu \sigma} T_{\nu \rho}+4 \delta_{\alpha \rho} \delta_{\beta \mu} T_{\nu \sigma}-4 \delta_{\alpha \mu} \delta_{\beta \rho} T_{\nu \sigma}+5 \delta_{\alpha \beta} \delta_{\mu \rho} T_{\nu \sigma}\right) .
\end{array}
$$

The expression on the left-hand side contains also $\mathcal{O}_{\mu \nu \rho \sigma}^{(4)}$, which is however orthogonal to the stress tensor and so does not contribute to $c$ and $a$.

We can define our $a$ in the following way. The scalar, spinor and vector OPE terms $(T T)^{T}$ are a basis for the OPE structure [26]. We use the stress-tensor two-point function and the $T T$ OPE to associate effective numbers $n_{s, f, v}$ of scalars, fermions and vectors to the spin-2 conformal field and then apply the free-fields formulas for $c$ and $a$.

We write

$$
\langle(T T) T\rangle=n_{s}\langle(T T) T\rangle_{s}+n_{f}\langle(T T) T\rangle_{f}+n_{v}\langle(T T) T\rangle_{v} .
$$

Here $(T T)$ means that we take the limit in which the distance between the first two $T$-insertions tends to zero, and so we can use the OPE calculated above. On the right-hand side, $\langle(T T) T\rangle_{s, f, v}$ denote the corresponding expressions for one free real scalar, one fermion and one vector, which can be read in [26]. Clearly, only the $T$-content of the OPE is relevant in the limit we are considering: $\langle(T T) T\rangle=(T T)^{T}\langle T T\rangle$, where $(T T)^{T}$ denotes the structure multiplying $T$ in the TT OPE. For example, $(T T)^{T}$ is the structure contained between the first square brackets in (4.23). We have

$$
c(T T)^{T}=\frac{1}{120}\left[n_{s}(T T)_{s}^{T}+6 n_{f}(T T)_{f}^{T}+12 n_{v}(T T)_{v}^{T}\right] .
$$

Using the two-point functions and OPEs of free fields [26] we arrive, by comparison, at

$$
n_{s}=0, \quad n_{f}=\frac{256}{27}, \quad n_{v}=\frac{64}{27} .
$$

Observe that $n_{s}=0$ can be inferred immediately from the OPE. Scalar fields produce a structure $(T T)_{s}^{T}$ with the maximal number of uncontracted $x_{\mu}$ 's (six), vector fields give a structure $(T T)_{v}^{T}$ with the minimum number (two) and $(T T)_{f}^{T}$, for the spinors, contain four uncontracted $x_{\mu}$ 's. A quick inspection of the propagator shows that our structure $(T T)^{T}$ cannot contain more than four uncontracted $x_{\mu}$ 's. 
The final result is

$$
c=\frac{32}{45}, \quad a=\frac{848}{1215}, \quad \frac{c-a}{c}=\frac{1}{54} .
$$

We see that both $c$ and $a$ are positive, as well as $n_{f}$ and $n_{v}$, and that $c$ is "almost" equal to $a$, but slightly greater.

The procedure used to calculate $c$ and $a(4.24)$ guarantees that these values parametrize the trace anomaly in the appropriate way. However, we cannot write for the trace anomaly a closed expression such as (1.1), which makes use of the coupling to external gravity, and we need to work always at the level of correlators and OPEs. It is meaningful, nevertheless, to truncate the right-hand side of (1.1) to the quadratic terms in an expansion of the gravitational field around flat space.

We have therefore shown that $c$ and $a$ can be appropriately defined in our theories despite the absence of a coupling to external gravity, and that they are positive. Some issues need to be better understood, for example the relation (if any) between the gauge-invariant tensor $T_{\mu \nu}$ and the Noether tensor.

The $\mathcal{O}_{\alpha \beta \gamma \delta}^{(4)}$-content of the OPE can be extracted with the replacement

$$
F_{\mu \nu \alpha}^{+} F_{\rho \sigma \beta}^{-}+F_{\mu \nu \alpha}^{-} F_{\rho \sigma \beta}^{+} \rightarrow \delta_{\nu \sigma} \mathcal{O}_{\alpha \beta \mu \rho}^{(4)}-\delta_{\nu \rho} \mathcal{O}_{\alpha \beta \mu \sigma}^{(4)}-\delta_{\mu \sigma} \mathcal{O}_{\alpha \beta \nu \rho}^{(4)}+\delta_{\mu \rho} \mathcal{O}_{\alpha \beta \nu \sigma}^{(4)} .
$$

The presence of this multiply-conserved, spin-4, dimension- 4 operator, absent in ordinary theories, is here emphasized, as a good illustration of the new features of higher-spin conformal field theory and the role of the multiple-conservation condition. The hope is that the ghost degrees of freedom, or spin- $s$ operators with dimension lower than $2+s$, might be controlled in some way. A sufficiently strong interaction might raise the dimensions of all operators. I recall that the Nachtmann theorem [8], in unitary theories, states that the anomalous dimensions of the higher-spin currents generated by the singular terms of the OPE are to some extent correlated [24, 25] (e.g. the anomalous dimensions increase with the spin and the magnitude of the interaction). It is conceivable that a similar result here would ensure that below a certain energy threshold, when the interaction is sufficiently strong, the theory is perfectly unitary, i.e. all spin- $s$ operators have dimension greater than or equal to $2+s$.

\subsection{Antisymmetric conformal tensors}

With antisymmetric tensors, many of the nice features of symmetric tensors disappear. In particular, conformal invariance spoils both the positivity of the action and gauge invariance. A 2-form $A_{\mu \nu}$ has the conformal-invariant action

$$
S=\frac{1}{2} \int\left[\left(\partial_{\alpha} A_{\mu \nu}\right)^{2}-4\left(\partial_{\alpha} A_{\alpha \nu}\right)^{2}\right]
$$

With $A_{\mu \nu}=\partial_{\mu} \zeta_{\nu}-\partial_{\nu} \zeta_{\mu}$ we find $S=-\frac{1}{2} \int\left(\partial_{\alpha} A_{\mu \nu}\right)^{2}$, so that the action is not positive-definite and gauge invariance is completely lost. The theory can be coupled in a (classically) conformal way to Abelian and non-Abelian gauge fields, as well as gravity. Renormalizable couplings to 
symmetric higher-spin conformal fields is instead problematic. For example, a coupling of a complex antisymmetric tensor with a spin-3 field of the Pauli type, such as

$$
i g F_{\mu \nu \alpha \beta} A_{\mu \alpha} \bar{A}_{\nu \beta}=\mathcal{O}_{\mu \nu \rho}^{(3)} \chi_{\mu \nu \rho}+\text { total derivatives }
$$

vanishes because of the cyclic identity.

The $A_{\mu \nu}$-field equations and propagator read

$$
\square A_{\mu \nu}-2 \partial_{\alpha}\left(\partial_{\mu} A_{\alpha \nu}+\partial_{\nu} A_{\mu \alpha}\right)=0, \quad\left\langle A_{\mu \nu}(x) A_{\rho \sigma}(0)\right\rangle=\frac{-1}{8 \pi^{2}|x|^{2}}\left(I_{\mu \rho}(x) I_{\nu \sigma}(x)-I_{\mu \sigma}(x) I_{\nu \rho}(x)\right) .
$$

Observe that the propagator is reflection-negative. We conclude that antisymmetric conformal tensor fields are much less interesting than the symmetric tensors.

\section{Conformal fermionic fields}

A spin- $(s+1 / 2)$ field is described by a spinor $\psi_{\mu_{1} \cdots \mu_{s}}$ with $s$ Lorentz indices, completely symmetric and traceless.

The transformation of the spinor under coordinate inversion is

$$
\psi_{\mu_{1} \cdots \mu_{s}} \rightarrow|x|^{2} \not \gamma \gamma_{5} I_{\mu_{1}}^{\nu_{1}}(x) \cdots I_{\mu_{s}}^{\nu_{s}}(x) \psi_{\nu_{1} \cdots \nu_{s}} .
$$

The contraction $\gamma_{\beta} \psi_{\beta \mu_{2} \cdots \mu_{s}}$ transforms as a spin- $(s-1 / 2)$ conformal spinor. Further contractions with gamma matrices are automatically zero, owing to complete tracelessness. Instead $\sum_{i=1}^{s} \gamma_{\mu_{i}} \gamma_{\alpha} \psi_{\alpha \mu_{1} \cdots \hat{\mu}_{i} \cdots \mu_{s}}$ transforms as a spin- $(s+1 / 2)$ spinor. Therefore we can always impose

$$
\gamma_{\alpha} \psi_{\alpha \mu_{2} \cdots \mu_{s}}=0
$$

and preserve conformal invariance. Under this condition the most general conformal lagrangian is simply

$$
\mathcal{L}=\bar{\psi}_{\mu_{1} \cdots \mu_{s}} \not \partial \psi_{\mu_{1} \cdots \mu_{s}},
$$

any other possible term vanishing because of (5.25). The proof that (5.26) transforms correctly is rather lengthy, but straightforward. To make (5.25) manifest, we can insert appropriate projectors:

$$
\begin{aligned}
\mathcal{L}= & \left(\bar{\psi}_{\mu_{1} \cdots \mu_{s}}-\frac{1}{2(s+1)} \sum_{i=1}^{s} \bar{\psi}_{\alpha \mu_{1} \cdots \hat{\mu}_{i} \cdots \mu_{s}} \gamma_{\alpha} \gamma_{\mu_{i}}\right) \not \partial\left(\psi_{\mu_{1} \cdots \mu_{s}}-\frac{1}{2(s+1)} \sum_{i=1}^{s} \gamma_{\mu_{i}} \gamma_{\alpha} \psi_{\alpha \mu_{1} \cdots \hat{\mu}_{i} \cdots \mu_{s}}\right)= \\
= & \bar{\psi}_{\mu_{1} \cdots \mu_{s}} \not \partial \psi_{\mu_{1} \cdots \mu_{s}}-\frac{s}{s+1} \bar{\psi}_{\alpha \mu_{2} \cdots \mu_{s}} \gamma_{\alpha} \partial_{\beta} \psi_{\beta \mu_{2} \cdots \mu_{s}}-\frac{s}{s+1} \bar{\psi}_{\alpha \mu_{2} \cdots \mu_{s}} \gamma_{\beta} \partial_{\alpha} \psi_{\beta \mu_{2} \cdots \mu_{s}} \\
& +\frac{s(s+2)}{2(s+1)^{2}} \bar{\psi}_{\alpha \mu_{2} \cdots \mu_{s}} \gamma_{\alpha} \not \partial \gamma_{\beta} \psi_{\beta \mu_{2} \cdots \mu_{s} .} .
\end{aligned}
$$

The field equations are

$$
\not \partial \psi_{\mu_{1} \cdots \mu_{s}}=\frac{1}{s+1} \sum_{i=1}^{s} \gamma_{\mu_{i}} \partial_{\alpha} \psi_{\alpha \mu_{1} \cdots \hat{\mu}_{i} \cdots \mu_{s}} .
$$


Condition (5.25) is not sufficient to eliminate the ghosts of the theory. We see that no gauge invariance survives and the theory can be straightforwardly coupled to Abelian and nonAbelian gauge fields, as well as gravity. In particular, $c$ and $a$ can be defined in the usual way. In the next section, I discuss the case $s=1$ in detail and compute the contribution of conformal spinors to the gauge beta function.

\subsection{Spin $3 / 2$}

For $s=1$ the action

$$
\begin{aligned}
S & =\int \mathcal{L}=\int \bar{\psi}_{\mu}\left[\not \partial \psi_{\mu}-\frac{1}{2} \gamma_{\alpha} \partial_{\mu} \psi_{\alpha}-\frac{1}{2} \gamma_{\mu} \partial_{\alpha} \psi_{\alpha}+\frac{3}{8} \gamma_{\mu} \not \partial \gamma_{\alpha} \psi_{\alpha}\right]= \\
& =\int \bar{\psi}_{\mu} P_{\mu \nu} \not P_{\nu \rho} \psi_{\rho}, \quad P_{\mu \nu}=\delta_{\mu \nu}-\frac{1}{4} \gamma_{\mu} \gamma_{\nu}
\end{aligned}
$$

is invariant under coordinate inversion, the field being transformed as

$$
\psi_{\mu} \rightarrow|x|^{2} \not \partial \gamma_{5} I_{\mu}^{\nu}(x) \psi_{\nu}
$$

The field equations are

$$
\not \partial \psi_{\mu}=\frac{1}{2} \gamma_{\mu} \partial \cdot \psi
$$

bearing in mind that $\gamma \cdot \psi=0$. The field equations imply also

$$
\left(\square \delta_{\mu \nu}-\partial_{\mu} \partial_{\nu}\right) \psi_{\nu}=0, \quad \not \partial \partial \cdot \psi=0 .
$$

The transversal component of $\psi_{\mu}$ obeys an ordinary wave equation, while $\partial \cdot \psi$ obeys the Dirac equation. The transformation $\delta \psi_{\mu}=\partial_{\mu} \epsilon$ is not a symmetry, however, since it preserves neither $\gamma \cdot \psi=0$ nor (5.27).

Our theory coincides with the theory called "singular" by Haberzett in the context of the nuclear theory of hadronic resonances: see formula (40) of ref. 17]. Its conformal invariance, and the unicity of the theory in this respect, is here emphasized.

I investigate in detail the coupling to Abelian and non-Abelian gauge fields, obtained by covariantizing the derivatives:

$$
\mathcal{L}=\frac{1}{4}\left(F_{\mu \nu}^{a}\right)^{2}+\bar{\psi}_{\mu}^{i}\left[\not D^{i j} \psi_{\mu}^{j}-\frac{1}{2} \gamma_{\alpha} D_{\mu}^{i j} \psi_{\alpha}^{j}-\frac{1}{2} \gamma_{\mu} D_{\alpha}^{i j} \psi_{\alpha}^{j}+\frac{3}{8} \gamma_{\mu} \not D^{j i} \gamma_{\alpha} \psi_{\alpha}\right]
$$

Here $a$ is the index of the fundamental representation of the gauge group $G$, and $i, j$ are indices of the matter representation $R$. The notation for the covariant derivative is $D_{\mu}^{i j} \psi_{\nu}^{j}=$ $\partial_{\mu} \psi_{\nu}^{i}+g\left(T^{a}\right)^{i j} A_{\mu}^{a} \psi_{\nu}^{j}$, as usual. The spin-3/2 propagator is

$$
\begin{aligned}
\left\langle\psi_{\mu}^{i}(k) \bar{\psi}_{\nu}^{j}(-k)\right\rangle & =-\frac{i \delta^{i j}}{k^{2}}\left[\not k \delta_{\mu \nu}-k_{\mu} \gamma_{\nu}-k_{\nu} \gamma_{\mu}+\frac{1}{2} \gamma_{\mu} \not k \gamma_{\nu}+\frac{2}{k^{2}} k_{\mu} \not k k_{\nu}\right] \\
& =-\frac{i \delta^{i j}}{k^{2}} P_{\mu \alpha} \not k\left(\delta_{\alpha \beta}+2 \frac{k_{\alpha} k_{\beta}}{k^{2}}\right) P_{\beta \nu}
\end{aligned}
$$


and the vertex is

$$
\left\langle\psi_{\mu}^{i} \bar{\psi}_{\nu}^{j} A_{\rho}^{a}\right\rangle=-g T_{i j}^{a} P_{\mu \alpha} \gamma_{\rho} P_{\alpha \nu}
$$

The theory is conformal at the classical level, and scale invariance is broken, as usual, by the radiative corrections at the quantum level. I have computed the one-loop beta function of this model in two different ways (gluon self-energy and three-gluon vertex), with the result

$$
\beta(g)=-\frac{g^{3}}{48 \pi^{2}}\left[11 C(G)-20 C\left(R_{3 / 2}\right)-4 C\left(R_{1 / 2}\right)\right] .
$$

The correction due to our spin-3/2 field is the term proportional to $C\left(R_{3 / 2}\right)$, while the term proportional to $C\left(R_{1 / 2}\right)$ is the usual spin-1/2 contribution, here inserted for comparison.

We see that this peculiar type of "matter" contributes to the beta function with the same sign as ordinary matter. For $C\left(R_{3 / 2}\right) \lesssim \frac{11}{20} C(G)$ the one-loop beta function is arbitrarily small with respect to the higher-order corrections, which allow us to conclude that there is a nontrivial IR fixed point, trustable in perturbation theory, and a conformal window, which is the main reason why these theories are an interesting laboratory of models for the ideas of [1]. Similar arguments extend to arbitrary half-integer spin. Note that our theories, in spite of their non-unitarity, are renormalizable and are not higher-derivative. For this reason we do not compare our results, for example, with the supergravity calculations, which cannot give evidence of a conformal window.

\section{Conclusions}

We have explored higher-derivative theories and higher-spin conformal theories, and studied the central charges $c$ and $a$, their positivity properties, the existence of renormalizable couplings and conformal windows. These theories are good toy models for investigations in the spirit of [1, 5], the study of questions concerning quantum irreversibility and the search for a unified description of higher- and lower-spin fields. Antisymmetric conformal tensors and higher-derivative theories exhibit severe violations of positive-definiteness and reflection positivity. Instead, there is evidence that higher-spin conformal symmetric tensors and fermions have positive $c$ and $a$. The symmetric tensors, moreover, have a positive-definite action and a peculiar gauge symmetry. These properties, I believe, make higher-spin conformal theories worthy of attention, even if they are not unitary.

Acknowledgement. I am grateful to U. Aglietti, F. Bastianelli, H.B. Nielsen, R. Rattazzi and A. Waldron for discussions.

\section{References}

[1] D. Anselmi, Exact results about quantum field theories interpolating between pairs of conformal field theories, XIV International Workshop on High-Energy Physics and Quantum Field Theory, Moscow, June 1999, hep-th/9910255. 
[2] D. Anselmi, D.Z. Freedman, M.T. Grisaru and A.A. Johansen, Nonperturbative formulas for central functions in supersymmetric theories, Nucl. Phys. B 526 (1998) 543 and hepth/9708042.

[3] D. Anselmi, Anomalies, unitarity and quantum irreversibility, Ann. Phys. (NY) 276 (1999) 361 and hep-th/9903059.

[4] D. Anselmi, Quantum irreversibility in arbitrary dimension, Nucl. Phys. B 567 (2000) 331 and hep-th/9905005.

[5] D. Anselmi, Towards the classification of conformal field theories in arbitrary dimension, Phys. Lett. B 476 (2000) 182. hep-th/9908014.

[6] S.M. Christensen and M.J. Duff, New gravitational index theorems and super theorems, Nucl. Phys. B 154 (1979) 301; Axial and conformal anomalies for arbitrary spin in gravity and supergravity, Phys. Lett. B 76 (1978) 571.

[7] P. van Nieuwenhuizen, On ghost-free tensor lagrangians and linearized gravitation, Nucl. Phys. B 60 (1973) 478.

[8] O. Nachtmann, Positivity constraints for anomalous dimensions, Nucl. Phys. B 63 (1973) 237.

[9] S. Ferrara, R. Gatto and A. Grillo, Positivity constraints on anomalous dimensions, Phys. Rev. D 9 (1974) 3564.

[10] M.A. Vasiliev, Higher-spin gauge theories in four, three and two dimensions, Int. J. Mod. Phys. D5 (1996) 763 and hep-th/9611024.

[11] D. Anselmi, J. Erlich, D.Z. Freedman and A.A. Johansen, Positivity constraints on anomalies in supersymmetric gauge theories, Phys. Rev. D57 (1998) 7570 and hep-th/9711035.

[12] F. Bayen, Thèse, Université de Dijon (1970).

[13] M.S. Drew and J.D. Gegenberg, Conformally covariant massless spin-two field equations, Nuovo Cim. A 60 (1980) 41.

[14] S. Deser and R.I. Nepomechie, Anomalous propagation of gauge fields in conformally flat spaces, Phys. Lett. B 132 (1983) 321.

[15] A.O. Barut and B. Xu, On conformally covariant spin-2 and spin-3/2 equations, J. Phys. A: Math. Gén. 15 (1982) L207.

[16] C. Delfino Galles, A conformal gauge for the spin-2 field, Nuovo Cim. Lett. 42 (1085) 382.

[17] H. Haberzett, Propagation of a massive spin-3/2 particle, nucl-th/9812043. 
[18] V. Pascalutsa and R. Tiemmermans, Field theory of nucleon to higher-spin baryon transitions, Phys. Rev. C 60 (1999) 042201 and nucl-th/9905065.

[19] T. Appelquist and J. Carazzone, Infrared singularities and massive fields, Phys. Rev. D 11 (1975) 2856.

[20] R.J. Riegert, A non-local action for the trace anomaly, Phys. Lett. B 134 (1984) 56.

[21] I. Antoniadis and E. Mottola, 4-D quantum gravity in the conformal sector, Phys. Rev. D45 (1992) 2013.

I. Antoniadis, P.O. Mazur and E. Mottola, Criticality and scaling in 4D quantum gravity, Phys. Lett. B 394 (1997) 49 and hep-th/9611145.

[22] S.D. Odintsov, Curved space-time formulation of the conformal sector of $4 \mathrm{D}$ quantum gravity, Z. Phys. C 54 (1992) 531.

[23] A. Pais and G.E. Uhlenbeck, On field theories with non-localized action, Phys. Rev. 79 (1950) 145.

[24] D. Anselmi, The N=4 quantum conformal algebra, Nucl. Phys. B 541 (1999) 369 and hep-th/9809192.

[25] D. Anselmi, Quantum conformal algebras and closed conformal field theory, Nucl. Phys. B 554 (1999) 415 and hep-th/9811149.

[26] D. Anselmi, Theory of higher spin tensor currents and central charges, Nucl. Phys. B 541 (1999) 323 and hep-th/9808004.

[27] D. Anselmi, Higher-spin current multiplets in operator-product expansions, Class. and Quantum Grav. 17 (2000) 1383 and hep-th/9906167. 